\title{
Ethan J. Halpner (ed): Clinical cardiac CT
}

\author{
Thieme, New York, 2008. ISBN: 978-1-58890-618-2
}

\author{
Roberto De Rosa • Luigi Mansi
}

Published online: 15 July 2009

(C) Springer-Verlag 2009

In recent years there has been a revolution in cardiovascular imaging following the development of new techniques such as cardiac CT. The publication of a comprehensive text written by one of the leaders in the field is therefore an important contribution, as it should fill an immediate need.

The aim of the book is to provide the means to confidently perform and accurately interpret cardiac CT, as the author wrote in her brief preface, "provides a didactic tutorial to optimize CT technique as well as CT case material that reflects the full spectrum of normal variation and pathologic findings". The author has for the most part succeeded in this aim. This book should serve as a valuable resource for residents and fellows, and for those not yet routinely involved in cardiovascular CT imaging, and is a good text for quick and easy reference in daily practice for experienced cardiac imagers.

The text consists of two sections. The first offers the obligatory condensed overview of cardiovascular CT imaging, providing information on the key issues and the
CT principles and anatomical information to perform cardiac CT. The fundamentals of cardiovascular CT imaging are explained with essential protocol data to remember and information on normal coronary anatomy and anatomic variation.

In the second part, in which an atlas accompanies the text, are described the full spectrum of pathologic findings that are seen in adult cardiology practice. A conclusion at the end of every paragraph gives the opportunity for the reader to reevaluate in real time his or her knowledge. All illustrations, which are mainly high-quality CT images of clinical cases, supplement and enhance the quality of the text, but, although they are of fundamental value, their copiousness occasionally makes it difficult to maintain continuity in reading. But this very minor problem is completely counterbalanced by the completeness of the information provided.

In summary, the book is an important reference for beginners and experienced radiologists, and provides all the information that will be useful to any specialist who already performs or is interested in performing cardiovascular $\mathrm{CT}$.

R. De Rosa $(\bowtie)$

Ospedale San Gennaro,

Napoli, Italy

e-mail: robertoderosa@libero.it

L. Mansi

Seconda Università di Napoli,

Napoli, Italy 